\title{
Anastral spindle assembly and $\gamma$-tubulin in Drosophila oocytes
}

\author{
Sharyn A Endow*, Mark A Hallen
}

\begin{abstract}
Background: Anastral spindles assemble by a mechanism that involves microtubule nucleation and growth from chromatin. It is still uncertain whether $\gamma$-tubulin, a microtubule nucleator essential for mitotic spindle assembly and maintenance, plays a role. Not only is the requirement for $\gamma$-tubulin to form anastral Drosophila oocyte meiosis I spindles controversial, but its presence in oocyte meiosis I spindles has not been demonstrated and is uncertain.

Results: We show, for the first time, using a bright GFP fusion protein and live imaging, that the Drosophila maternally-expressed $\gamma T$ Tub37C is present at low levels in oocyte meiosis I spindles. Despite this, we find that formation of bipolar meiosis I spindles does not require functional $\gamma$ Tub37C, extending previous findings by others. Fluorescence photobleaching assays show rapid recovery of $\gamma$ Tub37C in the meiosis I spindle, similar to the cytoplasm, indicating weak binding by $\gamma$ Tub37C to spindles, and fits of a new, potentially more accurate model for fluorescence recovery yield kinetic parameters consistent with transient, diffusional binding.

Conclusions: The FRAP results, together with its mutant effects late in meiosis I, indicate that $\gamma$ Tub37C may perform a role subsequent to metaphase I, rather than nucleating microtubules for meiosis I spindle formation. Weak binding to the meiosis I spindle could stabilize pre-existing microtubules or position $\gamma$-tubulin for function during meiosis II spindle assembly, which follows rapidly upon oocyte activation and completion of the meiosis I division.
\end{abstract}

\section{Background}

Anastral spindles assemble without centrosomes by a pathway that is still not fully understood. In particular, the mechanism by which microtubule nucleation occurs has not been well defined. Although chromatin has been shown to play an essential role [1], the involvement of the microtubule nucleator, $\gamma$-tubulin, is still an open question. $\gamma$-Tubulin localizes to centrosomes and other microtubule organizing centers in mitosis and is essential for nucleating microtubules in organisms as diverse as yeast, Drosophila, Xenopus, humans, and higher plants [2-5]. $\gamma$-Tubulin is also found on spindle microtubules, where it has been proposed to nucleate microtubules for spindle maintenance by functioning in a chromatinmediated nucleation pathway that augments the dominant pathway of nucleation by centrosomes [6,7].

$\gamma$-Tubulin is present in cells as a large ring complex, $\gamma$ TuRC, comprising 12-13 $\gamma$-tubulin molecules associated

\footnotetext{
* Correspondence: endow@duke.edu

Department of Cell Biology and Structural Biology \& Biophysics Program, Duke University
}

C Biomed Central

(c) 2011 Endow and Hallen; licensee BioMed Central Ltd. This is an Open Access article distributed under the terms of the Creative Commons Attribution License (http://creativecommons.org/licenses/by/2.0), which permits unrestricted use, distribution, and reproduction in any medium, provided the original work is properly cited. with as many as $\sim 7-8$ other proteins $[8,9] . \gamma$ TuRC forms from a small complex, $\gamma$ TuSC, consisting of $\gamma$-tubulin bound to two other proteins [10-12]. The mechanism by could serve as a ring-like template for a 12 -or 13 protofilament microtubule $[13,14]$ or, alternatively, $\alpha / \beta$ tubulin dimers could assemble onto an end of the large ring complex to form individual protofilaments [15].

Although $\gamma$-tubulin is believed to play a central role in mitotic spindle assembly and maintenance in many organisms, its role in anastral spindles that lack centrosomes is less certain. Analysis of mutants that affect the Drosophila oocyte- and early embryo-specific $\gamma$ Tub37C [16] has led to the conclusion that $\gamma$-tubulin plays an essential role in nucleating microtubules for anastral oocyte meiosis I (MI) spindle assembly [17]. Conflictingly, another study of the same mutants concluded that $\gamma$-tubulin is not required for oocyte MI spindle formation [18]. Moreover, attempts by several groups to stain oocyte MI spindles using anti- $\gamma$ Tub37C antibodies have which $\gamma \mathrm{TuRC}$ nucleates microtubules is uncertain - it 
produced negative results $[17,19,20]$, raising doubts as to whether $\gamma$-tubulin is even present in the spindle.

Failure to localize $\gamma$-tubulin to MI spindles might be due to inadequate permeabilization of the dense cytoplasm of fixed Drosophila oocytes, hindering antibody staining, rather than the absence of $\gamma$-tubulin in spindles. A way around this problem is to analyze oocytes of flies expressing $\gamma$-tubulin tagged with an easily detectable fluorescent marker. Here we show using transgenic flies expressing $\gamma$ Tub37C fused to a bright green fluorescent protein (GFP) that $\gamma$-tubulin is present at low levels in oocyte MI spindles. Nonetheless, bipolar MI spindles are present in $\gamma$-tubulin mutant oocytes, indicating that $\gamma$-tubulin is not required to form oocyte MI spindles. Fluorescence recovery after photobleaching (FRAP) assays demonstrate that $\gamma$-tubulin binding interactions with the spindle are weak and transient. $\gamma$-Tubulin is thus not required for MI spindle formation and binds weakly to the spindle; this may be important in stabilizing pre-existing microtubules or positioning $\gamma$-tubulin for function subsequent to MI spindle assembly.

\section{Results}

\section{$\gamma$ Tub37C localizes to oocyte MI spindles}

Females expressing $\gamma$ Tub37C fused to a bright GFP, regulated by the kinesin-14 ncd oocyte- and early embryo-specific promoter [21] were analyzed for this study. The females also expressed endogenous $\gamma$ Tub37C without GFP, which is maternally loaded into the oocytes like Ncd. Western blot analysis showed lower levels of $\gamma$ Tub37C-GFP than endogenous $\gamma$ Tub37C in both adult females and ovaries (Additional file 1 Figure S1). Quantitation of the cross-reacting bands gave a ratio of 0.4-0.5 for $\gamma$ Tub37C-GFP to $\gamma$ Tub37C, indicating that the level of $\gamma \mathrm{Tub3} 3 \mathrm{C}-\mathrm{GFP}$ in the transgenic flies is approximately half that of the endogenous $\gamma$ Tub37C. This extends our previous estimate of the expression level of $\gamma$ Tub37C-GFP in early embryos by measurement of GFP fluorescence, which we found to be similar to Ncd-GFP regulated by the same promoter [21]. Thus, $\gamma$ Tub37C-GFP is not highly over-expressed, as frequently occurs when transgenes are driven by gal4 or other inducible promoters.

Live oocytes were imaged to determine whether $\gamma$ tubulin is present in the MI spindle. Many, but not all of the oocytes showed detectable levels of $\gamma$-tubulin-GFP in late stage $13(\mathrm{n}=16$, total $=19)$ or stage $14(\mathrm{n}=9$, total $=11)$ spindles. $\gamma$-Tubulin localized differently to the spindles than Ncd-mRFP1, co-expressed by the females $(n=10)$ as a spindle marker [22]. It was typically found on the thick spindle equator, or spindle midzone, in late stage 13 oocytes and at the spindle poles, including the pole bodies, in stage 14 oocytes (Figure 1A). Its absence from spindles of some oocytes was attributed to the low levels of $\gamma$-tubulin in the spindle and the difficulty of detecting the protein. In addition, the endogenous unlabeled $\gamma$ Tub37C in the oocytes probably occupies binding sites in the spindle, reducing the $\gamma$ Tub37C-GFP fluorescence.

Oocytes of females $(n=7)$ expressing $\gamma$ Tub37C-GFP, but not Ncd-mRFP1, were also examined to exclude the possibility of imaging artefacts caused by Ncd-mRFP1. $\gamma$-Tubulin was again found at low levels in late stage 13 $(\mathrm{n}=5$, total $=11)$ or stage $14(\mathrm{n}=17$, total $=18) \mathrm{MI}$ spindles (Figure 1C), demonstrating detectable spindle localization even in the absence of another fluorescent protein in the spindle. The images of $\gamma T u b 37 C-g f p$ oocyte spindles provide the first direct evidence that $\gamma$ tubulin is present in the Drosophila oocyte MI spindle. The low levels in the spindle and the difficulty in permeabilizing oocytes for antibody staining explain why previously reported antibody localization experiments did not produce positive results.

\section{YTub37C mutant embryos exhibit bipolar MI spindles}

We then examined $\gamma$ Tub37C APL10 mutant oocytes expressing Ncd-GFP as a spindle marker [23] to determine whether normal MI spindles were present. YTub37C APL10 mutant females lay eggs, but the eggs fail to hatch due to early embryo lethality [24]; the lethality is fully penetrant and can be used as indicator of APL10 homozygosity. We confirmed this by mating females in single pairs and monitoring vials for matings that produced embryos but no larvae, then extracting DNA from the female carcasses for PCR and $\gamma$ Tub37C DNA sequence analysis after ovaries were removed for oocyte spindle imaging.

Fifteen females produced embryos none of which hatched; bipolar spindles $(\mathrm{n}=30$, total $=32)$ were observed in oocytes from 10 females (Figure 2). Five females did not exhibit GFP-labeled spindles because the $n c d-g f p$ transgene, which was on a chromosome that was segregating in the stock, was absent, as confirmed by PCR. Eleven of the 15 females were homozygous for APL10 by sequence analysis; two females gave poor sequence due to the small amount of DNA that was recovered and two females that showed no GFP spindle labeling were not sequenced. As a control, a female that produced embryos that hatched into larvae was sequenced and found to be APL10/+. Early embryo lethality of $\gamma$ Tub37C APL10 is thus fully penetrant, confirming earlier reports $[18,24]$. Despite this, APL10 mutant oocytes exhibited bipolar MI spindles, many of which appeared normal $(\mathrm{n}=14)$, although some spindles had split poles $(n=8)$ or were frayed $(n=6)$ (Figure 2, arrow), or both $(\mathrm{n}=2)$. A few spindles were not bipolar $(\mathrm{n}=2)$, resembling those of severe loss-of-function $n c d$ mutants, which are defective in assembly [20]. 


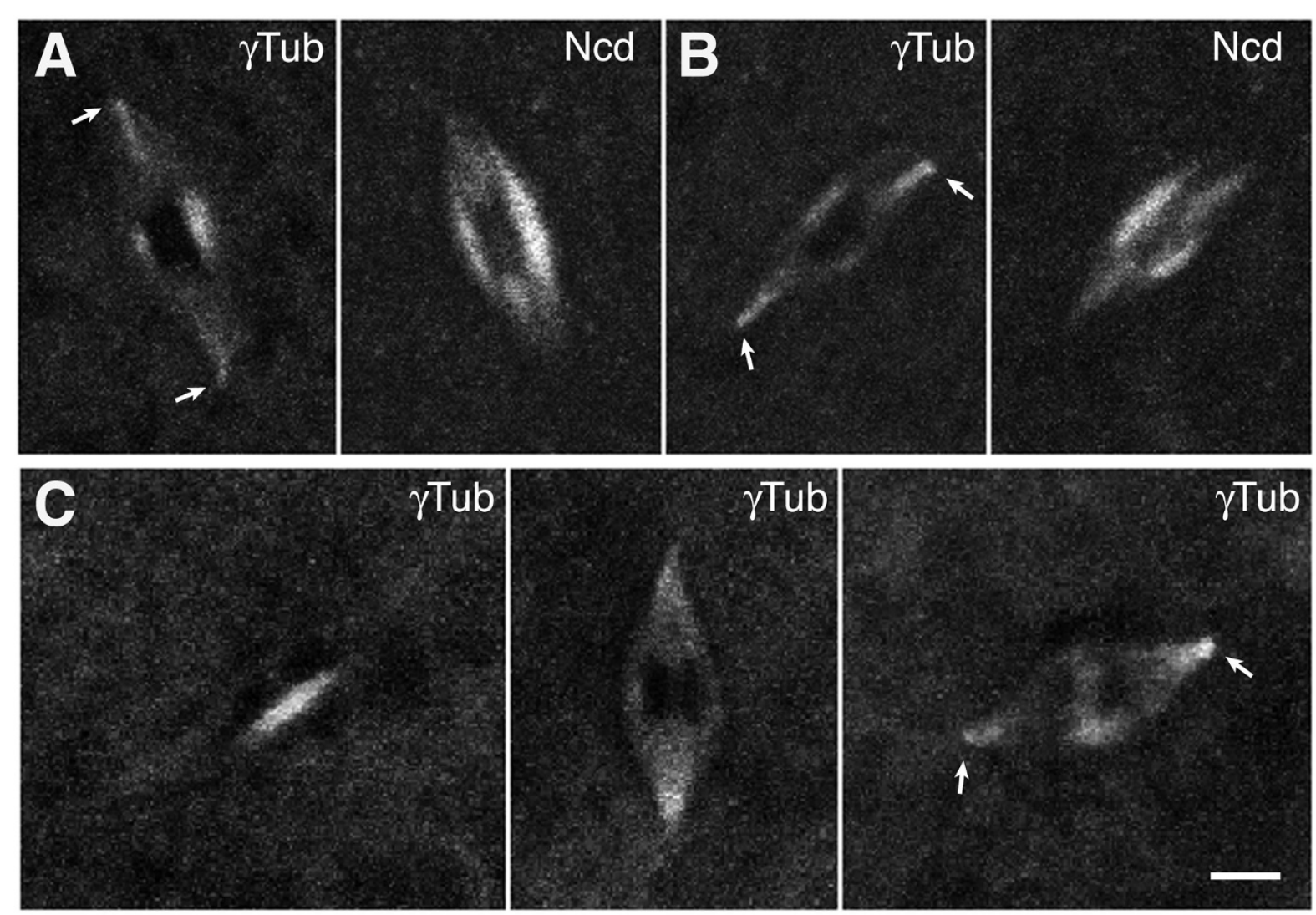

Figure $1 \gamma$-Tubulin localizes to oocyte MI spindles. (A) MI spindle of a late stage 13 or (B) stage 14 oocyte expressing $\gamma$ Tub37C-GFP ( $\gamma$ Tub, left) and Ncd-mRFP1 (Ncd, right). (C) Ml spindles of a late stage 13 oocyte (left) or stage 14 oocytes (middle, right) expressing $\gamma$ Tub37C-GFP but not Ncd-mRFP1. $\gamma$-Tubulin is present in the Ml spindle midzone and at the spindle poles, including pole bodies (arrows). Bar, $3 \mu \mathrm{m}$.

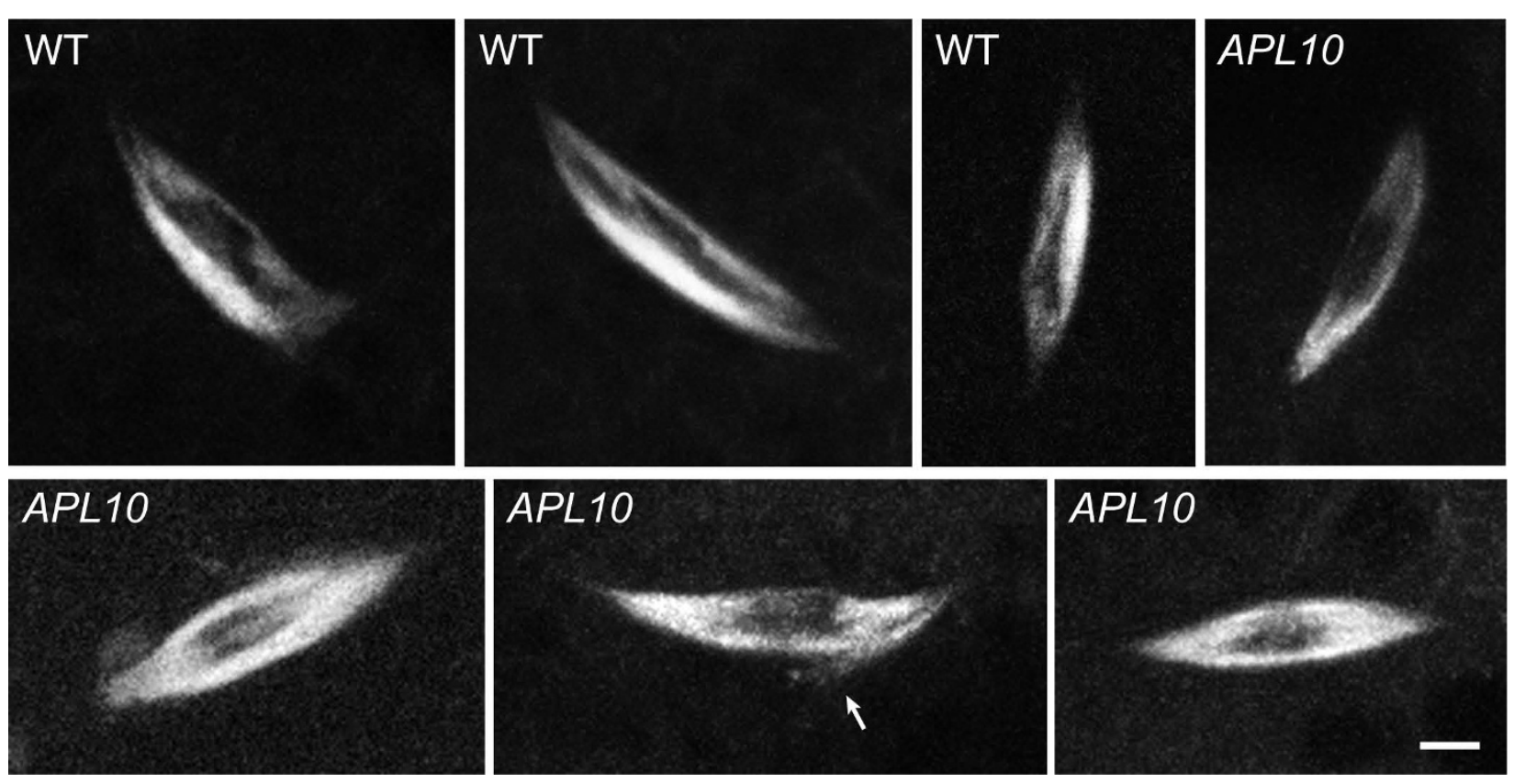

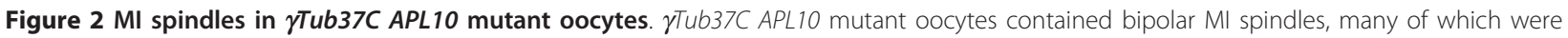
normal, resembling those in wild-type (WT) oocytes, although some were frayed (arrow) or had split poles (not shown). Spindles visualized by Ncd-GFP. Bar, $3 \mu \mathrm{m}$. 
Some of the abnormal MI spindles could have arisen because of insufficient Ncd, given that approximately a fourth of the fifteen $\gamma$ Tub37C APL10 females were expected to be homozygous for the $n c d$-gfp transgene needed to image the spindles and the ncd null mutant $c a^{\text {nd }}$, and two copies of the transgene do not completely rescue $c a^{\text {nd }}[21-23,25]$. However, all ten $\gamma$ Tub37C APL10 females with GFP-labeled spindles appeared to carry a wild-type $n c d^{+}$gene by PCR analysis, and thus should have been wild-type for $n c d$ [20], producing infrequent Ncd-related spindle abnormalities (see below). Despite this, all ten females yielded one or more oocytes with an abnormal spindle.

Split poles or frayed spindles can also appear in wildtype oocytes - a previous study of wild-type ncd-gfp oocytes showed normal bipolar MI spindles ( $\mathrm{n}=15$, total $=17$ ) with a few spurred or multipolar spindles $(n=2)$ [26]. Some of these spindle abnormalities arise when imaging MI spindles in the final stages of assembly while the chromosomes are still undergoing movements [20] or due to inadvertent activation of oocytes while preparing them for imaging. However, the high frequency of abnormal $\gamma$ Tub37C APL10 spindles $(\mathrm{n}=18$, total $=32)$ and the nature of the abnormalities (split poles, frayed spindles, defective assembly) raise the possibility that $\gamma$-tubulin may play a role in stabilizing MI spindles during assembly or ensuring correct spindle morphology.

Nonetheless, the presence of a significant proportion of normal-appearing bipolar MI spindles in APL10 mutant oocytes $(\sim 44 \%)$ indicates that $\gamma$ Tub37C does not appear to be essential for the assembly of bipolar MI spindles. This conclusion was also reached in an earlier study [18], which reported that all the $\gamma T u b 37 C$ mutants examined, including the null mutant, assembled bipolar oocyte spindles. However, this study, as well as other previous studies, did not demonstrate the presence of $\gamma$ tubulin in the MI spindle, as noted above.

\section{A mutant $\gamma$ Tub37C does not bind to $\mathrm{MI}$ spindles}

Several $\gamma T u b 37 C$ mutants have been reported, including the APL10 allele [24] used in this study. These mutants cause female sterility due to early embryo lethality, providing strong evidence that the mutated residues are required for $\gamma$ Tub37C function. The alleles that have been sequenced, including APL10 (E117K) [27], affect $\gamma$ tubulin residues that are conserved from fly to human. We tested the cytological effects of mutating a conserved $\gamma$ Tub37C residue by recovering transgenic flies expressing an APL10-like mutation, E116R-gfp, immediately adjacent to E117, which is mutated in APL10. Both E116 and E117 are conserved in human $\gamma$-tubulin as E116 and D117, and are present in $\gamma$ Tub37C helix H3, a helix that is thought to form lateral interactions between $\gamma$-tubulin molecules in $\gamma$ TuRC [28].
Live oocytes of $\gamma T u b 37 C^{+}$females $(\mathrm{n}=6)$ expressing $\gamma$ Tub37C E116R-GFP together with Ncd-mRFP1 to visualize MI spindles showed GFP fluorescence dispersed throughout the cytoplasm, but no GFP localized to late stage $13(n=5)$ or stage 14 spindles $(n=11)$ (Figure 3 ). The dark region in the center of the spindle corresponds to the condensed chromosomes, which exclude fluorescence [23]. The dark region was detected in $\gamma$ Tub37C E116R-gfp oocytes (Figure 3, arrows), but $\gamma$ Tub37C E116R-GFP was not observed in spindles above cytoplasmic levels, in contrast to $\gamma$ Tub37C-GFP, which was specifically localized to the MI spindle.

To estimate the expression level of the mutant relative to wild-type protein, $\gamma$ Tub37C E116R-GFP cytoplasmic fluorescence was normalized to the dark chromosomal region and compared to $\gamma$ Tub37C-GFP cytoplasmic fluorescence normalized in the same way. This analysis showed comparable cytoplasmic fluorescence for $\gamma$ Tub37C E116R-GFP (1.51 \pm 0.03 a.u., $\mathrm{n}=6)$ and $\gamma$ Tub37C-GFP (1.31 \pm 0.04 a.u., $\mathrm{n}=6)$. Western blot analysis also showed similar levels of $\gamma$ Tub37C E116RGFP and $\gamma$ Tub37C-GFP compared to $\gamma$ Tub37C in ovaries (Additional file 1 Figure S1). Thus, the mutant

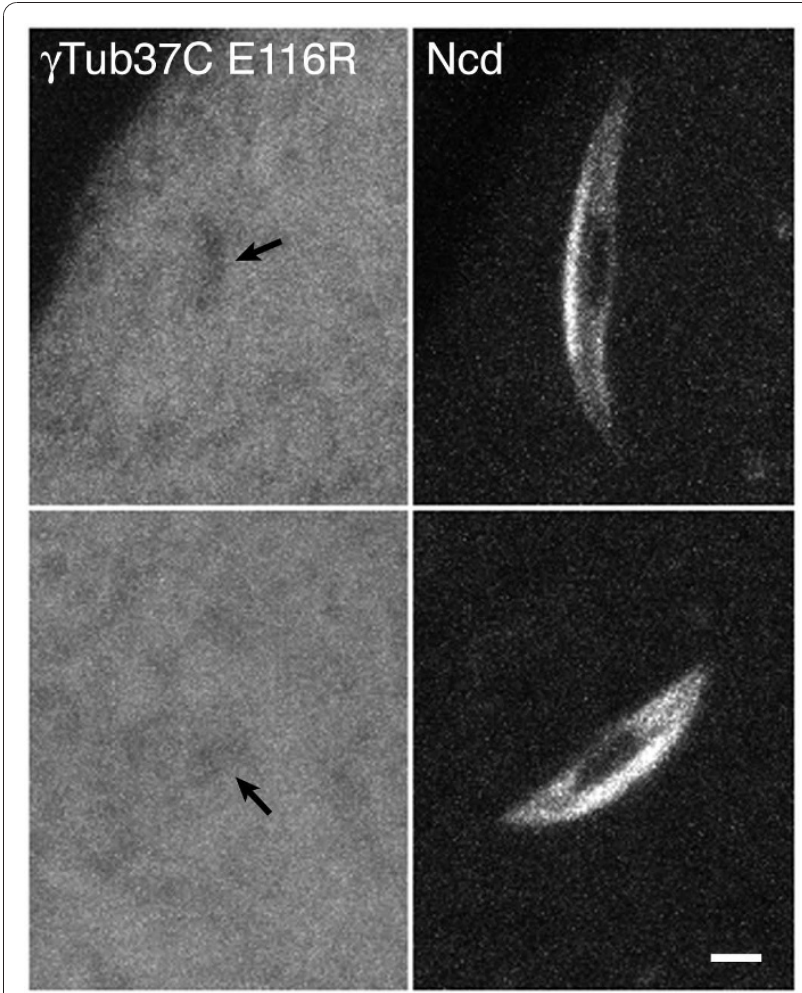

Figure 3 Mutant $\gamma$ Tub37C in oocytes. $\gamma$ Tub37C E116R-GFP, mutated in a conserved $\gamma$ Tub37C residue, is present in the cytoplasm, but does not localize to oocyte Ml spindles (left). Dark regions (arrows) correspond to the condensed $\mathrm{Ml}$ chromosomes [23]. Spindles detected with Ncd-mRFP1 (right). Bar, $3 \mu \mathrm{m}$. 
protein is expressed and is present in the cytoplasm at levels similar to the wild-type protein; despite this, it does not localize to MI spindles. The ability to detect $\gamma$ Tub37C-GFP but not $\gamma$ Tub37C E116R-GFP in oocyte MI spindles indicates that $\gamma$ Tub37C binds specifically although at low levels to the spindle midzone and poles, rather than being present in spindles simply because it diffuses into the spindle from the cytoplasm. It also shows that mutating a conserved residue perturbs the localization of $\gamma \mathrm{Tub} 37 \mathrm{C}$ to the spindle, possibly due to the failure to form higher-order $\gamma$-tubulin complexes.

Although the $\gamma$ Tub37C APL10 mutation, E117K, differs from the $\gamma$ Tub37C E116R mutant that we tested cytologically, it is similar in that it also affects a conserved, negatively charged residue in helix $\mathrm{H} 3$ and changes it to a positively charged residue. This means that APL10 could have a similar effect to $\gamma \mathrm{Tub37C}$ E116R in potentially disrupting $\gamma$-TuRC formation or other $\gamma$ Tub37C interactions. Thus, despite the likelihood that the APL10-mutated $\gamma$ Tub37C is not associated with the MI spindle, bipolar spindles can form, as observed in APL10 mutant oocytes. These observations indicate that assembly of bipolar MI spindles does not require functional $\gamma$ Tub37C.

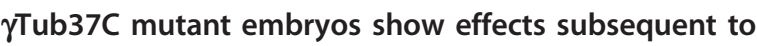 metaphase I}

$\gamma$ Tub37C APL10 mutant embryos were examined to determine the earliest stage at which $\gamma$ Tub37C is required for function. Embryos, collected for $\geq 1.5-2 \mathrm{hr}$, were fixed and stained with rhodamine- $\alpha$-tubulin antibody and DAPI to image the spindles and chromosomes, respectively. Ncd-GFP, co-expressed by the mutant females, was also used to visualize the spindles; in some cases, spindles were imaged only with NcdGFP. Ore R embryos, collected for 0-15 min, were fixed and stained for controls.

Mutant embryos displayed complex abnormal spindle and chromosome configurations (Figure 4). Many embryos were blocked in stages that resembled late MI $(\mathrm{n}=4$, total $=13)$ or MII $(\mathrm{n}=5)$. The earliest stage of arrest observed was anaphase I (Figure 4A). The spindles and chromosomes in a given embryo frequently differed from one another in stage, e.g., a polar body in one embryo was associated with condensed MI-like chromosomes. Multiple anastral spindles containing metaphase II-like chromosomes were present in others (Figure 4B), instead of the typical MII spindle of wild type, which comprises two tandem spindles joined by a central spindle pole body (Figure 4E, top).

Polar bodies in several embryos contained $>2 \mathrm{~N}$ chromosomes, indicating failure to undergo the meiotic divisions or continued chromosome divisions after MII. One embryo displayed a single large astral spindle associated with a haploid set of chromosomes (Figure 4C), which we interpreted to be a cycle 1 mitotic spindle from an embryo that had failed to undergo pronuclear fusion, by comparison with wild type (Figure 4E, middle). Another embryo exhibited many small astral spindles (Figure 4D) that were a third to half the size of wild-type spindles at approximately the same cleavage cycle (Figure $4 \mathrm{E}$, bottom). The centrosomes associated with these spindles were presumably from the fertilizing sperm, but the small size of the spindles was consistent with their containing haploid, rather than diploid chromosome complements [29]. Spindles and chromosomes in many embryos had divided out of synchrony with each other, resulting in abnormal configurations that were difficult to interpret with certainty.

Overall, the earliest stage of arrest appeared to be anaphase I. Normal MII spindles were not observed and probably failed to form. Polar bodies could form even when the chromosomes had not completed the meiotic divisions. In some embryos, divisions occurred on multiple anastral spindles; in another, presumed haploid chromosome complements segregated on astral spindles. These observations are consistent with the interpretation that the earliest $\gamma$ Tub37C APL10 mutant defects occur in late MI and normal MII divisions fail to occur, and that pronuclear migration and fusion may also be defective. These findings extend the conclusion reached by others in a previous study that $\gamma T u b 37 C$ null mutant embryos may be arrested in meiosis [18].

\section{rTub37C binds weakly to MI spindles}

$\gamma$-Tubulin binding interactions with oocyte MI spindles were analyzed by performing FRAP assays and fitting models for fluorescence recovery to the data to obtain values for binding and dissociation rate constants and the diffusion coefficient. A large or small region of interest (ROI) was bleached in a $\gamma T u b 37 C$-gfp oocyte MI spindle or the adjacent cytoplasm, and fluorescence recovery was monitored in the bleach spot [30]. The data were corrected for photobleaching during recovery imaging by adding back the fluorescence that was lost in an unbleached region of the spindle or cytoplasm. The recovery curves for the spindle and cytoplasm were similar to one another with slightly faster recovery for the spindle than cytoplasm (Figure 5). The small ROI in both the spindle and cytoplasm showed faster recovery than the large ROI, indicating that diffusion plays a dominant role in recovery.

The corrected data were fit to FRAP models to estimate kinetic parameters for $\gamma$-tubulin binding to the oocyte spindle or cytoplasm. The models tested were a diffusion phase-binding phase (previously referred to as diffusion-binding) and two-state binding dominant model [31], and a new conical reaction-diffusion model [32]. 


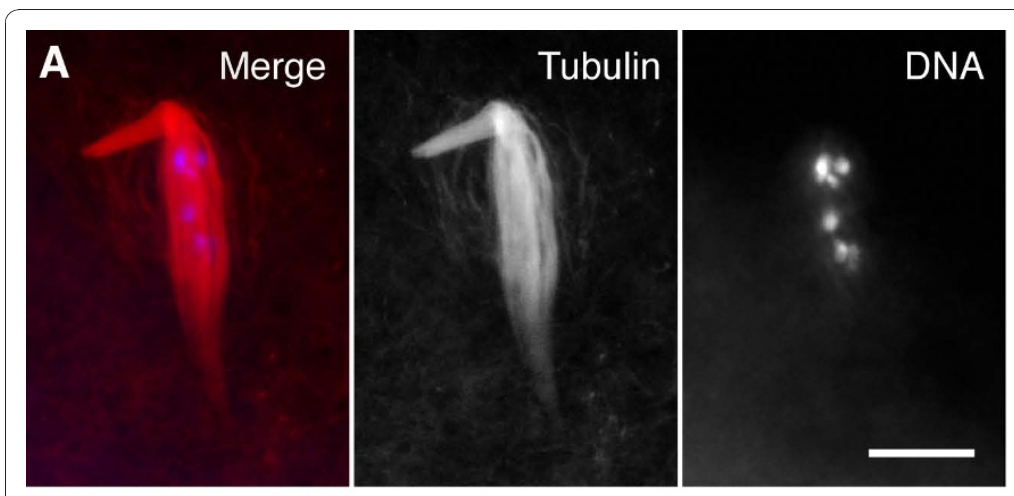

B

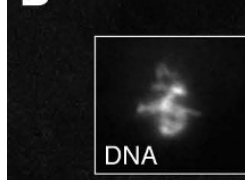

DNA

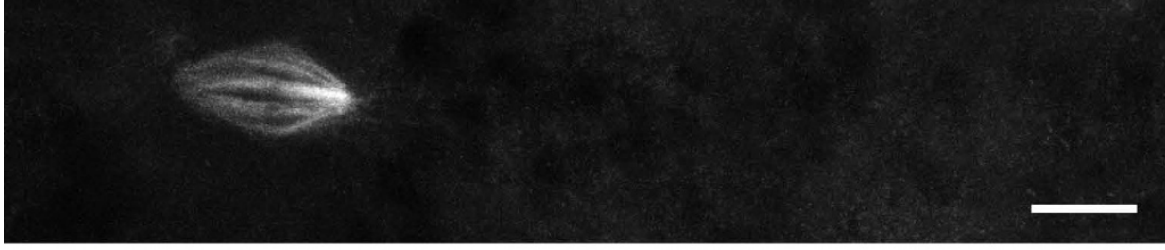

C

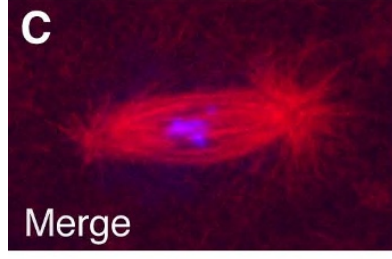

D Merge
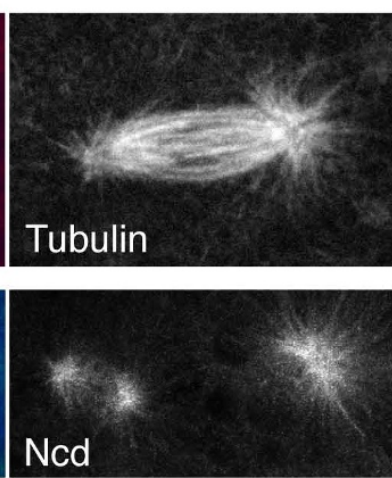
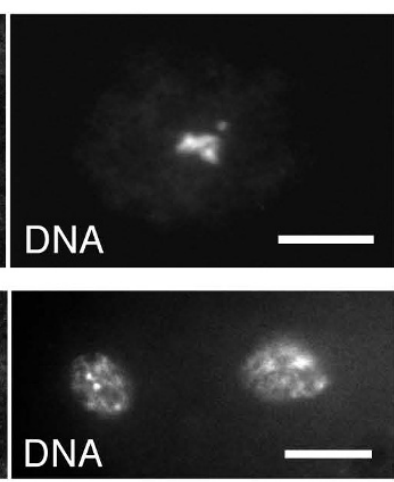
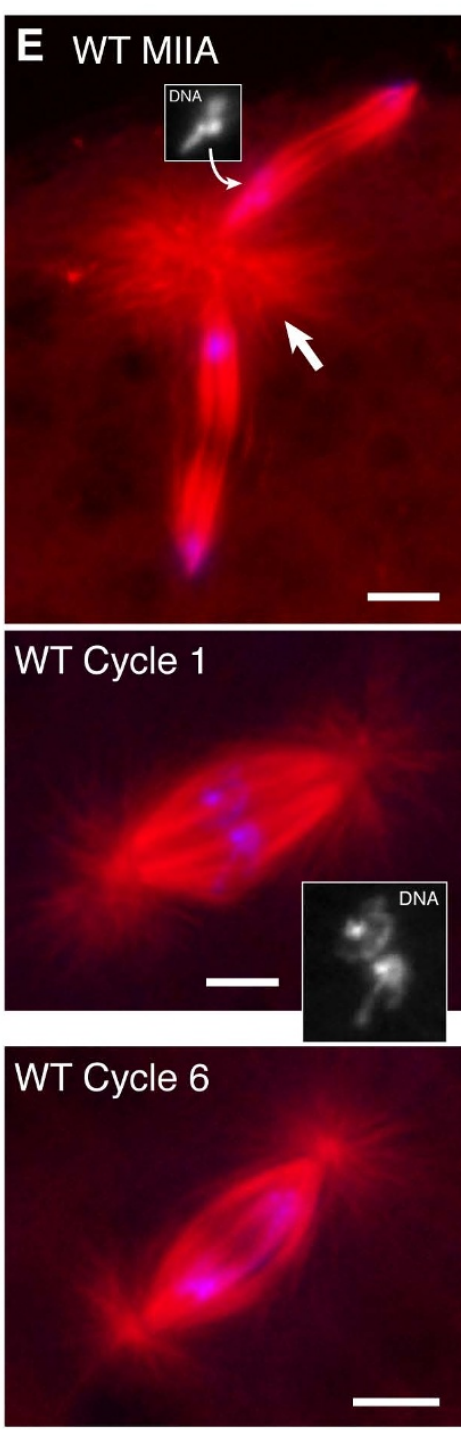

Figure 4 YTub37C APL10 mutant embryos. (A) Anaphase I arrest. (B) Anastral spindles associated with MII metaphase chromosomes (inset) from an embryo containing 6 or more anastral spindles and a polar body with $1 \mathrm{~N}$ chromosomes. (C) Astral cycle 1 mitotic spindle containing a haploid set of chromosomes. (D) Two small astral spindles associated with interphase or prometaphase nuclei from an embryo containing 20 astral spindles ( cycle 6) and two pairs of MII-like anastral spindles without central spindle bodies. (E) Wild-type spindles. Top, anaphase II spindle with central spindle pole body (large arrow) and segregating haploid chromosome sets (inset); middle, cycle 1 mitotic spindle containing two haploid chromosome sets (inset); bottom, cycle 6 mitotic spindle. Tubulin, red; Ncd-GFP, green; DNA, blue. Bars, 5 m.

The diffusion phase-binding phase and two-state binding dominant models assume a cylindrical bleach profile, which is a reasonable approximation when recovery shows a binding-dominant phase that can be used to estimate kinetic parameters for binding interactions with cellular structures [21]. The new conical reaction-diffusion model assumes a double-cone bleach profile that more closely resembles the actual point spread function of a high numerical aperture objective like the one used in this study. The model represents an exact analytical solution using Fourier and Hankel transforms for fluorescence recovery in the bleach region that is expected to yield more accurate estimates of binding and diffusion parameters than previous models.

Visually good fits for all three models were obtained for the MI spindle and cytoplasm FRAP data by concurrently fitting the data for the large and small ROIs [21]. A comparison of the kinetic parameters from the models is given in Additional file 1 Table S1. Values from the new conical reaction-diffusion model for the oocyte spindle and cytoplasm data are also given below and in Table 1; we believe that they are likely to be the most 


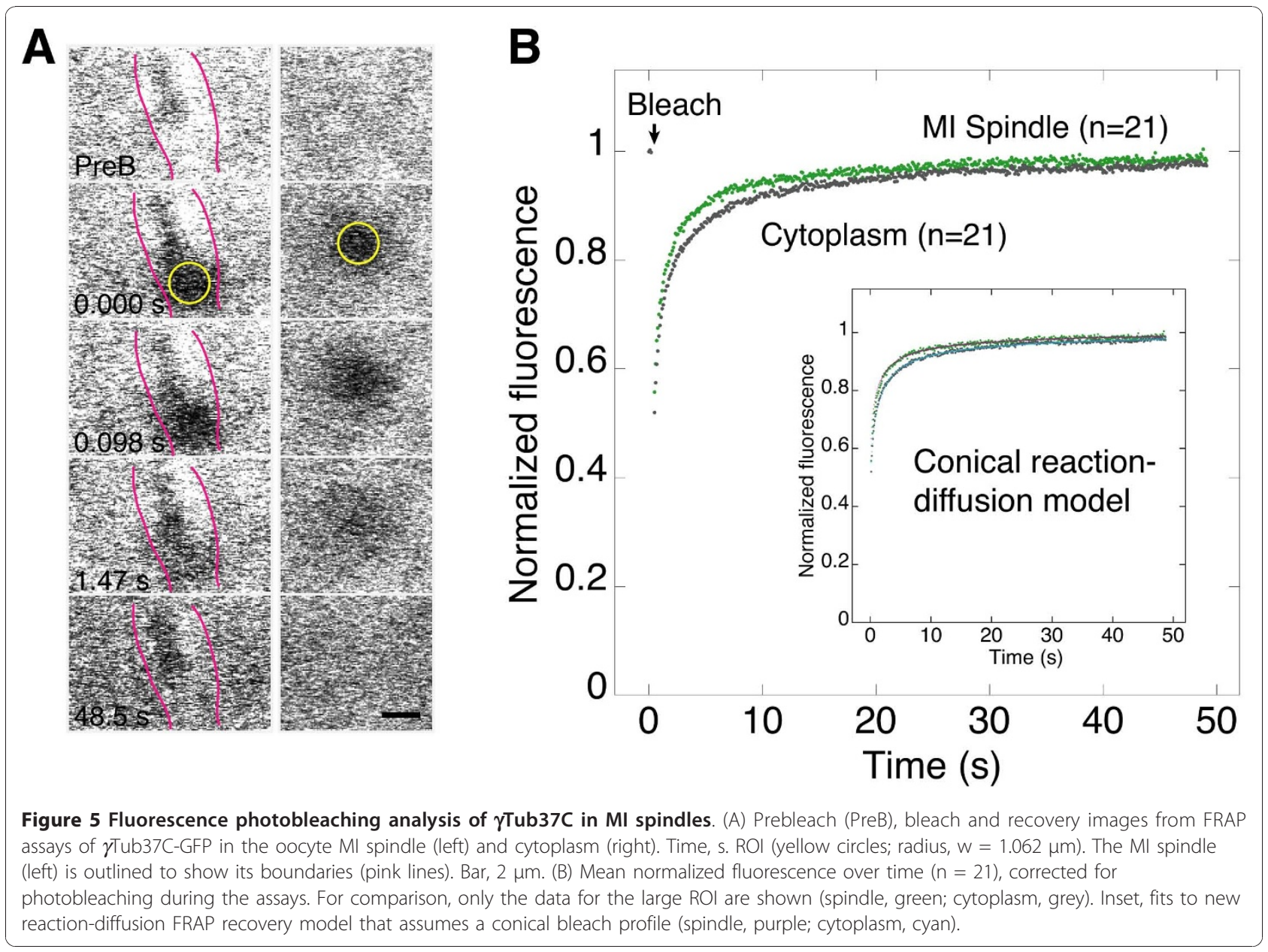

accurate of the three models because the model assumes a more accurate photobleaching profile and because of the dominant role of diffusion in the recovery.

For $\gamma$ Tub37C in the MI spindle, the diffusion coefficient, $\mathrm{D}=12 \pm 5 \mathrm{\mu m}^{2} / \mathrm{s}$; pseudo first-order binding constant, $\mathrm{k}_{\text {on }}^{*}=0.02 \pm 0.02 \mathrm{~s}^{-1}$; and dissociation constant,

Table 1 TTub37C kinetic parameters in the spindle and cytoplasm

\begin{tabular}{lccccc}
\hline $\begin{array}{l}\text { Spindle or } \\
\text { cytoplasm }\end{array}$ & $\begin{array}{c}\mathbf{D}\left(\mu \mathbf{m}^{2} /\right. \\
\mathbf{s})\end{array}$ & $\mathbf{k}_{\text {on }}^{*}\left(\mathbf{s}^{-1}\right)$ & $\mathbf{k}_{\text {off }}\left(\mathbf{s}^{-1}\right)$ & $\begin{array}{c}\mathbf{t}^{1} / 2 \\
(\mathbf{s})\end{array}$ & $\mathbf{C}_{\text {eq }}$ \\
\hline Ml spindle & $12 \pm 5$ & $0.02 \pm 0.02$ & $0.6 \pm 0.3$ & 1.1 & $0.03 \pm$ \\
& & & & 0.03 \\
Ooplasm & $8 \pm 3$ & $0.14 \pm 0.03$ & $0.9 \pm 0.1$ & 0.8 & $0.14 \pm$ \\
& & & & & 0.03 \\
Mitotic spindle & $28 \pm 10$ & $0.015 \pm$ & $0.23 \pm$ & 3.0 & $0.06 \pm$ \\
& & 0.006 & 0.04 & & 0.02 \\
Embryo & $26 \pm 8$ & $0.009 \pm$ & $0.15 \pm$ & 4.5 & $0.05 \pm$ \\
cytoplasm & & 0.004 & 0.03 & & 0.02 \\
\hline
\end{tabular}

Kinetic parameters from fits to the FRAP data of the conical reaction-diffusion model [32]; the half-time of recovery was calculated by $t^{1} / 2=\ln 2 / \mathrm{k}_{\text {off }}$ and the bound protein fraction at equilibrium by $C_{\mathrm{eq}}=\mathrm{k}^{*}{ }_{\mathrm{on}} /\left(\mathrm{k}^{*}{ }_{\mathrm{on}}+\mathrm{k}_{\mathrm{off}}\right)$ [31]. $\mathrm{k}_{\text {off }}=0.6 \pm 0.3 \mathrm{~s}^{-1}$, giving the recovery half-time, $\mathrm{t}^{1 / 2}=$ $1.1 \mathrm{~s}$, and bound protein fraction at equilibrium, $\mathrm{C}_{\mathrm{eq}}=$ $0.03 \pm 0.03$ (Table 1 ). In the oocyte cytoplasm or ooplasm, $\mathrm{D}=8 \pm 3 \mu \mathrm{m}^{2} / \mathrm{s}, \mathrm{k}^{*}$ on $=0.14 \pm 0.03 \mathrm{~s}^{-1}$ and $\mathrm{k}_{\text {off }}=0.9 \pm 0.1 \mathrm{~s}^{-1}$, giving $\mathrm{t}^{1} / 2=0.8 \mathrm{~s}$ and $\mathrm{C}_{\mathrm{eq}}=0.14 \pm$ 0.03 . Thus, the bound state is somewhat more prevalent in the ooplasm than in the meiotic spindle, although the majority of protein is still freely diffusing. Binding to the MI spindle is very low. The diffusion coefficients for the MI spindle and ooplasm are not significantly different; the lower diffusion coefficients than for $\gamma$ Tub37C in mitotic spindles and cytoplasm (see below and Table 1) reflect the more viscous ooplasm.

For comparison with the MI spindle results, we also fit the new conical reaction-diffusion model to our previous FRAP data for $\gamma$ Tub37C in early embryo mitotic spindles [21] (Table 1). The parameters differed from those we obtained previously by fitting the data to a two-dimensional recovery model, but were of the same order of magnitude and were in agreement with our previous conclusions. In the mitotic spindle, $\mathrm{D}=28 \pm$ $10 \mu \mathrm{m}^{2} / \mathrm{s}$ while $\mathrm{k}_{\text {on }}^{*}=0.015 \pm 0.006 \mathrm{~s}^{-1}$ and $\mathrm{k}_{\text {off }}=0.23$ 
$\pm 0.04 \mathrm{~s}^{-1}$, giving $\mathrm{t}^{1} / 2=3.0 \mathrm{~s}$ and $\mathrm{C}_{\mathrm{eq}}=0.06 \pm 0.02$. In the embryo cytoplasm, $\mathrm{D}=26 \pm 8 \mu \mathrm{m}^{2} / \mathrm{s}$ while $\mathrm{k}^{*}$ on $=$ $0.009 \pm 0.004 \mathrm{~s}^{-1}$ and $\mathrm{k}_{\text {off }}=0.15 \pm 0.03 \mathrm{~s}^{-1}$, giving $\mathrm{t}^{1} / 2=$ $4.5 \mathrm{~s}$ and $\mathrm{C}_{\mathrm{eq}}=0.05 \pm 0.02$. Thus, binding was relatively weak in both the mitotic spindle and embryo cytoplasm, with a similar turnover time and the same small amount of protein bound. The diffusion coefficient did not differ significantly between the mitotic spindle and embryo cytoplasm, as we observed for the MI spindle and ooplasm.

The kinetic parameters from the FRAP data indicate that $\gamma$ Tub37C binding to the MI spindle is transient with weak and diffusional properties, as in the ooplasm, similar to early embryo mitotic spindles and cytoplasm. This differs from the tight binding by $\gamma$ Tub37C to centrosomes of mitotic spindles [21], where it functions to nucleate microtubules for spindle formation. The diffusion coefficient for $\gamma$ Tub37C-GFP in the oocyte spindle of $\mathrm{D}=12 \pm 5 \mu \mathrm{m}^{2} / \mathrm{s}$ is probably too high to be $\gamma \mathrm{TuRC}$, which we estimated to have a D value of $3-8 \mu \mathrm{m}^{2} / \mathrm{s}$ in the embryo cytoplasm [21]; the D value in the oocyte spindle is expected to be even lower than this estimate because of the high viscosity of the ooplasm.

\section{Discussion}

Microtubule nucleation for anastral spindle assembly is dependent on chromatin, differing from astral spindles; however, the mechanism of nucleation is still not certain. The initial stages in formation of anastral Drosophila oocyte MI spindles have been studied by imaging live oocytes injected with rhodamine-tubulin [33] or expressing the kinesin-14 Ncd motor fused to GFP to visualize the spindle [20]. Microtubule growth from the condensed MI chromosomes or karyosome was observed in both cases, providing evidence that microtubule nucleation occurs from chromatin, as first observed in Xenopus extract spindles [1]. Time-lapse images obtained with Ncd-GFP showed small fluorescent foci or asters that migrated towards and associated with the karyosome, after which spindle microtubules grew out in all directions from the chromosomes [20]. The asters were also observed labeled with rhodaminetubulin but were thought not to play a role in spindle assembly, in part because they were not associated with spindle poles [33]. Their labeling by both Ncd-GFP and rhodamine-tubulin and behavior at early times following nuclear envelope breakdown are consistent with the interpretation that they consist of Ncd bound to small microtubules that nucleate microtubule growth after association with the chromosomes. Similar foci have been observed in early stages of spindle assembly in mouse oocytes [34].

Although the Ncd asters were interpreted as providing the nucleating activity for microtubule growth and MI spindle formation [20], it has still been a possibility that $\gamma$-tubulin, the microtubule nucleator for mitotic spindle assembly, plays a role. Two highly divergent $\gamma$-tubulin isoforms, $\gamma$ Tub23C and $\gamma$ Tub37C, exist in Drosophila. The two isoforms show $83 \%$ amino acid identity and are differentially expressed: $\gamma$ Tub23C is found in both males and females, but is not present in nurse cells or oocytes and is observed in early embryos only after cellularization, whereas $\gamma \mathrm{Tub} 37 \mathrm{C}$ is expressed only in females and accumulates in oocytes and early syncytially dividing embryos [16]. $\gamma$ Tub37C, the Drosophila oocyte- and early embryo-specific $\gamma$-tubulin, has not previously been localized to oocyte MI spindles, but we show here that it is present at low levels in the spindle midzone and on the poles and pole bodies, differing from the kinesin-14 motor, Ncd, which is present throughout the spindle. Its distribution indicates that $\gamma$-tubulin binds specifically to the MI spindle, rather than simply diffusing into the spindle from the ooplasm, which is expected to result in failure to accumulate in the spindle, as observed for a $\gamma$ Tub37C E116R mutant protein.

Despite its localization to the spindle, $\gamma$-tubulin shows transient binding interactions with the MI spindle by FRAP analysis. Fits of a new fluorescence recovery model, which is expected to yield more accurate values for binding parameters than previous models, gave a 30fold higher $\mathrm{k}_{\text {off }}$ than $\mathrm{k}^{*}$ on for $\gamma$ Tub37C binding to the MI spindle, indicating low binding affinity characterized by weak interactions. By comparison, the $\mathrm{k}_{\text {off }}$ was $\sim 6-$ fold greater than the $\mathrm{k}^{*}$ on for $\gamma \mathrm{Tub37C}$ in the ooplasm, again indicating weak binding interactions but somewhat higher binding affinity than in the spindle. The diffusion coefficient, D, for $\gamma$ Tub37C in the spindle did not differ significantly from the ooplasm.

The transient, weak binding by $\gamma$ Tub37C to the MI spindle differs from its tight binding to centrosomes of mitotic spindles in early embryos [21], but is similar to the weak binding by $\gamma \mathrm{Tub37C}$ to the mitotic spindle and cytoplasm of early embryos, which show a 15-17 fold higher $\mathrm{k}_{\text {off }}$ than $\mathrm{k}^{*}$ on. D for $\gamma$ Tub37C in the mitotic spindle and cytoplasm was the same, as for the MI spindle and ooplasm.

The weak binding by $\gamma$ Tub37C to MI spindles implies that it is not nucleating microtubules, given that it dissociates with a turnover rate that is too fast for substantial assembly to have occurred. Alternatively, it could nucleate microtubule growth by a mechanism that differs from that of centrosomes. However, the finding of bipolar MI spindles in $\gamma$ Tub37C APL10 mutant oocytes supports the interpretation that $\gamma \mathrm{Tub37C}$ is not required for MI spindle formation. Instead, microtubules for MI spindle formation are most likely nucleated by the Ncdbound asters observed to migrate to and associate with the karyosome [20]. 
$\gamma$ Tub37C is also present in oocyte MII spindles, where it is easily detectable by antibody staining, brightly labeling the central spindle pole body, which is thought to organize microtubules for rapid assembly of the two tandem MII spindles following anaphase I [35-37]. $\gamma$ Tubulin localization to the MII spindle is cell-cycle dependent - it is present in metaphase II but absent by late telophase II [35]. Although little is known about the recruitment of $\gamma$-tubulin to the MII spindle, the findings we report here suggest that weak binding by $\gamma$-tubulin to the MI spindle and its redistribution following anaphase I could account for its rapid appearance in the MII spindle following anaphase I. The MII spindle forms by reorganization of the anaphase I spindle without its disassembly - the central spindle pole body and new poles of the two tandem spindles form in the center of the anaphase I spindle [23]. Weak binding by $\gamma$ tubulin to the MI spindle midzone and poles may reflect the presence of interacting proteins that are positioned for function subsequent to anaphase I; their activation by phosphorylation or other modifications could account for the rapid sequence of events during progression of the oocyte MI and MII divisions.

Although the details of these events are likely to differ from organism to organism, basic features may be common to many organisms. For example, the finding of fluorescent asters in mouse oocytes that resemble those in Drosophila oocytes implies that the asters may be a general feature of "anastral" spindle formation. They were not reported in studies of Xenopus extract spindles, most likely because of the disruption of the cytoplasm during extract preparation. The asters are inferred from studies in Drosophila to consist of the kinesin-14 motor Ncd bound to short microtubules $[17,19,20]$; further studies are needed to characterize the asters and define their migration mechanism and role in microtubule nucleation.

\section{Conclusions}

$\gamma$-Tubulin does not appear to be essential for MI spindle assembly but binds weakly to the MI spindle, potentially stabilizing pre-existing microtubules or interacting with other proteins positioned for function later in the meiotic divisions. Available evidence suggests that $\mathrm{Ncd}$ motor-associated microtubule asters observed in previous studies are the primary source of new microtubules in the MI spindle. Further study of these asters should provide information regarding microtubule nucleation during "anastral" spindle assembly.

\section{Methods}

\section{Fly lines}

Transgenic flies expressing $\gamma$ Tub37C-GFP, $\gamma$ Tub37C E116R-GFP, Ncd-GFP and Ncd-mRFP1 have been reported previously $[21-23,25]$. One or two copies of $\gamma$ Tub37C-gfp fully rescues the female sterility of heterozygous $\gamma$ Tub37C APL10/DF(2L)VA23 females which carry the $\gamma$ Tub37C APL10 mutant allele over a deficiency that covers $\gamma$ Tub37C [21]. Thus, $\gamma$ Tub37C-gfp can complement a $\gamma \mathrm{Tub37C}$ mutant and appears to be functional. $\gamma$ Tub37C-GFP and $\gamma$ Tub37C E116R-GFP expression levels were estimated by Western blot analysis performed using conventional methods and total protein from $\gamma T u b 37 C-g f p$ females or ovaries crossreacted with anti- $\gamma$ Tub37C antibodies (a gift of Y. Zheng); $w^{1118}$ host females and ovaries were used as controls. Cross-reacting bands were quantitated using NIH ImageJ.

\section{Live imaging}

YTub37C APL10 mutant females were mated in single pairs and vials were monitored for embryo hatching up to 2-3 weeks. Ovaries were removed after several days of egg laying and remains of the females were quick frozen for DNA analysis. Live late stage 13 and stage 14 oocytes were prepared from the ovaries as described previously [20,30]; spindles were imaged by laserscanning confocal microscopy using a Bio-Rad Radiance2100 confocal scanhead mounted on a Zeiss Axioskop 2 Plus microscope, LaserSharp 2000 software, a 40x/1.3 NA Plan-NeoFluar oil immersion objective, and the 488-nm line of a $10 \mathrm{~mW} \mathrm{Kr}$-Ar laser. Co-expressed Ncd-GFP was used to visualize the spindles, which were analyzed for bipolarity and overall structure by comparison to spindles from control wild-type $n c d-g f p$ oocytes. Females that produced embryos that failed to hatch were confirmed homozygous for APL10 by PCR and DNA sequence analysis.

\section{Fixed embryos}

$\gamma$ Tub37C APL10 females were mated in single pairs to obtain embryos for analysis of mutant effects; vials were monitored for absence of embryo hatching to confirm the females were homozygous for APL10. Embryos were collected $1 \frac{1}{1} 2_{2}-2 \mathrm{hr}$; a few embryos that were analyzed were collected over longer time periods, either $4 \mathrm{hr}$ or overnight. Embryos were dechorionated manually in batches and incubated $\sim 2 \mathrm{~min}$ in heptane, washed in PBS and fixed for $\sim 3 \mathrm{~min}$ in 4-8\% formaldehyde in PBS, then washed and stored in PBS at $4{ }^{\circ} \mathrm{C}$. Vitelline membranes were removed manually prior to staining with rhodamine-labeled $\alpha$-tubulin antibody and/or DAPI. Spindles were imaged by rhodamine and/or coexpressed Ncd-GFP fluorescence using the Bio-Rad Radiance 2100 confocal microscope described above; chromosome images were acquired using a Hamamatsu Orca- $R^{2}$ cooled CCD camera attached to a Leitz Dialux 22 fluorescence microscope using a 63x/1.40 NA PL 
APO oil immersion objective. For controls, Ore R embryos collected 0-15 min were fixed and stained with rhodamine-labeled $\alpha$-tubulin antibody and DAPI.

\section{FRAP assays}

FRAP assays of $\gamma$ Tub37C-gfp F13F3 oocyte MI spindles and cytoplasm were performed at $\sim 22^{\circ} \mathrm{C}$ using a Zeiss LSM 510 confocal microscope and LSM 510 software, a 40x/1.3 NA Plan-NeoFluar oil immersion objective and the $488-\mathrm{nm}$ line of a $30 \mathrm{~mW}$ Ar laser set at $50 \%$ power, as described [30]. Assays, performed at zoom 3, consisted of three prebleach images, four rapid bleach scans at full laser power $($ ROI diameter $=29$ or 14 pixels; radius, $\mathrm{w}=$ 1.062 or $0.513 \mu \mathrm{m}$ at 13.653 pixels/ $\mu \mathrm{m}$ ) and 497 recovery images at $98 \mathrm{~ms} /$ image and low laser power (1.5\%). Fluorescence intensity in the large or small bleach spot over time recorded by the Zeiss 510 software was averaged for repeated assays $(n=21)$ of the same or different spindle or cytoplasm (3 oocytes from 3 females), except that the small ROI for the spindle consisted of fewer assays $(\mathrm{n}=10)$ of the spindle in 1 oocyte. Fluorescence loss during the assay due to photobleaching was measured in unbleached regions of the spindle or cytoplasm, averaged and added back to the mean recovery data to correct for photobleaching. After correction, the mean data for the large and small ROIs were fit to FRAP recovery models to estimate binding and dissociation rate constants for $\gamma$ Tub37C in the MI spindle and cytoplasm.

FRAP data for the spindle and cytoplasm in oocytes, acquired for the present study, and in early embryos, taken from a previous study [21], each with two different bleach spot sizes, were fit to fluorescence recovery models to determine binding and diffusion parameters. One of these was a new FRAP model that assumes a conical bleach profile approximating the point spread function of a high NA objective in a confocal microscope [32]. It has five parameters for each recovery curve: the diffusion coefficient D, pseudo-first-order association rate constant $\mathrm{k}^{*}$ on dissociation rate constant $\mathrm{k}_{\text {off }}$, bleach constant $\mathrm{k}_{\mathrm{b}}$ (which indicates the extent of bleaching), and total recovery $\mathrm{R}$ (which indicates the amount of fluorescence when recovery is complete and thus provides a normalization for the curve). This number of parameters is too many to extract accurately from a single recovery curve; a concurrent fitting strategy [21], in which parameters are shared between recovery curves, was thus used to allow accurate fitting.

The curve fitting consisted of two fits, one for the oocyte data and one for the early embryo data, since the assay conditions differed somewhat for the data sets and no parameters were assumed shared between the data sets. However, the large and small bleach spots in the spindle or cytoplasm were assumed to have the same values of $\mathrm{D}, \mathrm{k}^{*}$ on , and $\mathrm{k}_{\text {off }}$, since these are properties of $\gamma$ Tub37C in each cellular compartment and are not related to the bleaching. Furthermore, the large bleach spots in the spindle and cytoplasm were considered to share a value of $k_{b}$, as were the small bleach spots, although these values are different in the oocyte and early embryo data sets because the bleaching conditions differed somewhat. Each curve had its own value of R. Thus, each fit extracted twelve parameters from four data sets; a 3:1 parameter-to-data-set ratio is reasonable for nonlinear data like a FRAP recovery. The nlinfit function in MATLAB was used for the fitting, which was performed on the Duke University Blue Devil Grid. Because a greater amount of statistical error occurred in some data sets than others (for example, in smaller bleach spots), the robust option in nlinfit was used; this assigns greater weight to data with less error.

\section{Additional material}

Additional file 1: $\gamma$ Tub37C-GFP expression levels in transgenic flies and comparison of kinetic parameters from different FRAP models fit to the spindle and ooplasm data. Figure S1 shows a Western blot of wild-type and mutant $\gamma$ Tub37C-GFP expression levels in transgenic flies and ovaries compared to endogenous $\gamma$ Tub37C; Table S1 shows a comparison of $\gamma$ Tub37C kinetic parameters in the MI spindle and ooplasm derived from fits of different fluorescence recovery models to the FRAP data.

\section{Abbreviations}

FRAP: fluorescence recovery after photobleaching; GFP: green fluorescent protein; MI: meiosis I; MII: meiosis II; ROI: region of interest.

\section{Acknowledgements}

We thank V. Bennett for the use of a Zeiss LSM 510 confocal microscope, Y. Zheng and W. Sullivan for gifts of antibodies, and Duke University for use of the Blue Devil Grid computer cluster. This study was supported by grants to S.A.E. from the National Institutes of Health (R01 GM046225) and March of Dimes Foundation (\#1-FY-07-443). M.A.H. is a National Defense Science and Engineering Graduate Fellow and James B. Duke Scholar.

\section{Authors' contributions}

SAE designed and performed experiments, analyzed results and wrote the manuscript draft; MAH fit the FRAP data to models, interpreted and wrote the results, and helped revise the manuscript. Both authors read and approved the final manuscript.

Received: 4 October 2010 Accepted: 5 January 2011

Published: 5 January 2011

\section{References}

1. Heald R, Tournebize R, Blank T, Sandalzopoulos R, Becker P, Hyman A, Karsenti E: Self-organization of microtubules into bipolar spindles around artificial chromosomes in Xenopus egg extracts. Nature 1996, 382:420-425.

2. Oakley CE, Oakley BR: Identification of $y$-tubulin, a new member of the tubulin superfamily encoded by mipA gene of Aspergillus nidulans. Nature 1989, 338:662-664

3. Zheng $Y$, Jung MK, Oakley BR: $Y$-Tubulin is present in Drosophila melanogaster and Homo sapiens and is associated with the centrosome. Cell 1991, 65:817-823.

4. Liu B, Marc J, Joshi HC, Palevitz BA: A $\gamma$-tubulin-related protein associated with the microtubule arrays of higher plants in a cell cycle-dependent manner. J Cell Sci 1993, 104:1217-1228. 
5. Marshall LG, Jeng RL, Mulholland J, Stearns T: Analysis of Tub4p, a yeast $\gamma^{-}$ tubulin-like protein: implications for microtubule-organizing center function. J Cell Biol 1996, 134:443-454.

6. Lüders J, Patel UK, Stearns T: GCP-WD is a $y$-tubulin targeting factor required for centrosomal and chromatin-mediated microtubule nucleation. Nature Cell Biol 2006, 8:137-147.

7. Mahoney NM, Goshima G, Douglass AD, Vale RD: Making microtubules and mitotic spindles in cells without functional centrosomes. Curr Biol 2006, 16:564-569.

8. Hutchins JR, Toyoda Y, Hegemann B, Poser I, Hériché JK, Sykora MM, Augsburg M, Hudecz O, Buschhorn BA, Bulkescher J, Conrad C, Comartin D, Schleiffer A, Sarov M, Pozniakovsky A, Slabicki MM, Schloissnig S, Steinmacher I, Leuschner M, Ssykor A, Lawo S, Pelletier L, Stark H, Nasmyth K, Ellenberg J, Durbin R, Buchholz F, Mechtler K, Hyman AA, Peters JM: Systematic analysis of human protein complexes identifies chromosome segregation proteins. Science 2010, 328:593-599.

9. Teixidó-Travesa N, Villén J, Lacasa C, Bertran MT, Archinti M, Gygi SP, Caelles C, Roig J, Lüders J: The үTuRC revisited: a comparative analysis of interphase and mitotic human $Y$ TuRC redefines the set of core components and identifies the novel subunit GCP8. Mol Biol Cell 2010, 21:3963-3972.

10. Zheng $Y$, Wong ML, Alberts B, Mitchison T: Nucleation of microtubule assembly by a $\mathrm{\gamma}$-tubulin-containing ring complex. Nature 1995 , 378:578-583

11. Oegema K, Wiese C, Martin OC, Milligan RA, Iwamatsu A, Mitchison TJ, Zheng Y: Characterization of two related Drosophila $\mathrm{Y}$-tubulin complexes that differ in their ability to nucleate microtubules. J Cell Biol 1999, 144:721-733.

12. Xiong $Y$, Oakley BR: In vivo analysis of the functions of $Y$-tubulin-complex proteins. J Cell Sci 2009, 122:4218-4227.

13. Moritz M, Braunfeld M, Guénebaut V, Heuser J, Agard DA: Structure of the $\gamma$-tubulin ring complex: a template for microtubule nucleation. Nature Cell Biol 2000, 2:365-370.

14. Kollman JM, Polka JK, Zelter A, Davis TN, Agard DA: Microtubule nucleating Y-TuSC assembles structures with 13-fold microtubule-like symmetry. Nature 2010, 466:879-882

15. Erickson HP: $\gamma$-tubulin nucleation: template or protofilament? Nature Cell Biol 2000, 2:E93-E96

16. Wilson PG, Zheng Y, Oakley CE, Oakley BR, Borisy GG, Fuller MT: Differential expression of two $\mathrm{Y}$-tubulin isoforms during gametogenesis and development in Drosophila. Dev Biol 1997, 184:207-221.

17. Tavosanis G, Llamazares S, Goulielmos G, Gonzalez C: Essential role for $\gamma^{-}$ tubulin in the acentriolar female meiotic spindle of Drosophila. EMBO J 1997, 16:1809-1819.

18. Wilson PG, Borisy GG: Maternally expressed YTub37CD in Drosophila is differentially required for female meiosis and embryonic mitosis. Dev Biol 1998, 199:273-290

19. Matthies HJG, Miller RJ, Palfrey HC: Calmodulin binding to and CAMPdependent phosphorylation of kinesin light chains modulate kinesin ATPase activity. J Biol Chem 1993, 268:11176-11187.

20. Sköld HN, Komma DJ, Endow SA: Assembly pathway of the anastral Drosophila meiosis I oocyte spindle. J Cell Sci 2005, 118:1745-1755.

21. Hallen MA, Ho J, Yankel CD, Endow SA: Fluorescence recovery kinetic analysis of $\gamma$-tubulin binding to the mitotic spindle. Biophys J 2008, 95:3048-3058.

22. Hallen MA, Liang Z-Y, Endow SA: Ncd motor binding and transport in the spindle. J Cell Sci 2008, 121:3834-3841.

23. Endow SA, Komma DJ: Spindle dynamics during meiosis in Drosophila oocytes. J Cell Biol 1997, 137:1321-1336.

24. Schüpbach T, Wieshaus E: Female sterile mutations on the second chromosome of Drosophila melanogaster. I. Maternal effect mutations. Genetics 1989, 121:101-117.

25. Endow SA, Komma DJ: Centrosome and spindle function of the Drosophila Ncd microtubule motor visualized in live embryos using NcdGFP fusion proteins. J Cell Sci 1996, 109:2429-2442.

26. Sciambi CJ, Komma DJ, Sköld HN, Hirose K, Endow SA: A bidirectional kinesin motor in live Drosophila embryos. Traffic 2005, 6:1036-1046.

27. Wilson P: yTub37CD ${ }^{P L O}$. Personal communication to FlyBase 1999.

28. Aldaz H, Rice LM, Stearns T, Agard DA: Insights into microtubule nucleation from the crystal structure of human $\gamma$-tubulin. Nature 2005, 435:523-527.
29. Komma DJ, Endow SA: Haploidy and androgenesis in Drosophila. Proc Natl Acad Sci USA 1995, 92:11884-11888.

30. Liang Z-Y, Hallen MA, Endow SA: Mature Drosophila meiosis I spindles comprise microtubules of mixed polarity. Curr Biol 2009, 19:163-168,

31. Sprague BL, Pego RL, Stavreva DA, McNally JG: Analysis of binding reactions by fluorescence recovery after photobleaching. Biophys J 2004, 86:3473-3495.

32. Hallen MA, Layton AT: Expanding the scope of quantitative FRAP analysis. Journal of Theoretical Biology 2010, 262:295-305.

33. Matthies HJG, McDonald HB, Goldstein LSB, Theurkauf WE: Anastral meiotic spindle morphogenesis: role of the Non-Claret Disjunctional kinesin-like protein. J Cell Biol 1996, 134:455-464.

34. Schuh M, Ellenberg J: Self-organization of MTOCs replaces centrosome function during acentrosomal spindle assembly in live mouse oocytes. Cell 2007, 130:484-498.

35. Endow SA, Komma DJ: Assembly and dynamics of an anastral:astral spindle: the meiosis II spindle of Drosophila oocytes. J Cell Sci 1998, 111:2487-2495.

36. Riparbelli MG, Callaini $\mathrm{G}: \gamma$-Tubulin is transiently associated with the Drosophila oocyte meiotic apparatus. Eur J Cell Biol 1998, 75:21-28.

37. Brent AE, MacQueen A, Hazelrigg T: The Drosophila wispy gene is required for RNA localization and other microtubule-based events of meiosis and early embryogenesis. Genetics 2000, 154:1649-1662.

doi:10.1186/1471-2121-12-1

Cite this article as: Endow and Hallen: Anastral spindle assembly and $\gamma$ tubulin in Drosophila oocytes. BMC Cell Biology 2011 12:1.

\section{Submit your next manuscript to BioMed Central and take full advantage of:}

- Convenient online submission

- Thorough peer review

- No space constraints or color figure charges

- Immediate publication on acceptance

- Inclusion in PubMed, CAS, Scopus and Google Scholar

- Research which is freely available for redistribution
C Biomed Central 